\title{
Anisotropy and shear-layer edge dynamics of statistically unsteady, stratified turbulence
}

\author{
E. M. M. Wingstedt, ${ }^{1,2, a)}$ H. E. Fossum, ${ }^{1,2,3}$ B. A. Pettersson Reif, ${ }^{1,4}$ \\ and J. Werne ${ }^{5}$ \\ ${ }^{1}$ Norwegian Defence Research Establishment (FFI), P.O. Box 25, NO-2027 Kjeller, Norway \\ ${ }^{2}$ Department of Mathematics, University of Oslo, P.O. Box 1053 Blindern, \\ NO-0316 Oslo, Norway \\ ${ }^{3}$ Nammo Raufoss AS, P.O. Box 162, NO-2831 Raufoss, Norway \\ ${ }^{4}$ UNIK, P.O. Box 70, NO-2027 Kjeller, Norway \\ ${ }^{5}$ NorthWest Research Associates, CoRA Office, 3380 Mitchell Lane, Boulder, \\ Colorado 80301, USA
}

(Received 4 March 2015; accepted 26 May 2015; published online 9 June 2015)

\begin{abstract}
Direct numerical simulation data of an evolving Kelvin-Helmholtz instability have been analyzed in order to characterize the dynamic and kinematic response of shear-generated turbulent flow to imposed stable stratification. Particular emphasis was put on anisotropy and shear-layer edge dynamics in the net kinetic energy decay phase of the Kelvin-Helmholtz evolution. Results indicate a faster increase of small-scale anisotropy compared to large-scale anisotropy. Also, the anisotropy of thermal dissipation differs significantly from that of viscous dissipation. It is found that the Reynolds stress anisotropy increases up to a stratification level roughly corresponding to $\mathrm{Ri}_{g} \approx 0.4$, but subsequently decreases for higher levels of stratification, most likely due to relaminarization. Coherent large-scale turbulence structures are cylindrical in the center of the shear layer, whereas they become ellipsoidal in the strongly stratified edge-layer region. The structures of the Reynolds stresses are highly one-componental in the center and turn two-componental as stratification increases. Stratification affects all scales, but it seems to affect larger scales to a higher degree than smaller scales and thermal scales more strongly than momentum scales. The effect of strong stable stratification at the edge of the shear layer is highly reminiscent of the non-local pressure effects of solid walls. However, the kinematic blocking inherently associated with impermeable walls is not observed in the edge layer. Vertical momentum flux reversal is found in part of the shear layer. The roles of shear and buoyant production of turbulence kinetic energy are exchanged, and shear production is transferring energy into the mean flow field, which contributes to relaminarization. The change in dynamics near the edge of the shear layer has important implications for predictive turbulence model formulations. () 2015 AIP Publishing LLC. [http://dx.doi.org/10.1063/1.4922238]
\end{abstract}

\section{INTRODUCTION}

Shear-generated turbulence is a fundamental state of fluid flow that is present in the majority of real-life fluid dynamic systems. The ability of turbulence to mix momentum and scalar fields profoundly impacts the global flow field characteristics. Body forces arising from density variation due to, e.g., temperature or salinity differences, or the Coriolis force associated with an imposed rotation, may drastically change both the spatial and componental structures of the turbulence and thus also the overall behavior of the flow field. While the buoyancy forces associated with unstable stratification have a tendency to increase the turbulence intensity, stable stratification and a rapidly

\footnotetext{
a) Author to whom correspondence should be addressed. Electronic mail: Emma-My-Maria.Wingstedt@ffi.no.
} 
rotating frame of reference both have a tendency to suppress turbulence fluctuations and even cause relaminarization. In the latter case, both internal frictional losses and the mixing of momentum are substantially reduced. The implications are a significantly reduced rate of conversion of turbulence kinetic energy to internal energy (by the action of viscosity), reduced scale separation between small and large scale turbulence, and possibly increased large-scale anisotropy.

Transport and dispersion of passive contaminants are governed by turbulence and mean flow advection, rather than by molecular diffusion; the contaminant simply follows the large-scale three-dimensional and time-dependent velocity field. Passive contaminant transport is therefore expected to respond significantly to changes in the componental structure of the flow field caused by the imposition of a stably stratified background. An analogous situation occurs for dense-gas releases in an isothermal environment. In this case, the density variation is caused by the density difference between the contaminant itself and the ambient air.

Regardless of origin, spatial variation of fluid density generally affects all turbulence scales of the flow, changing the spatial and componental structure and in some cases even the rate of transfer of kinetic energy to internal energy. These particular features make stably stratified fluid flows challenging to model, both using statistically based turbulence closures and eddy-resolving simulations that rely on subgrid-scale models. The practical importance of stably stratified shear turbulence in general and its relevance to contaminant transport and dispersion constitute the primary motivations for the present study.

The componental structure of the flow is most commonly characterized by considering the anisotropy of the turbulence, e.g., the departure from an equipartitioning of turbulence energy in the three spatial directions. Several quantities can be derived and examined in order to analyze the anisotropy of a turbulent flow. In the work of Smyth and Moum, ${ }^{1}$ anisotropy tensors are derived from the Reynolds stress tensor and the viscous dissipation rate tensor, among others. Investigation of the anisotropy tensor components, the invariants, and the principal axes of these anisotropy tensors is conducted to shed light on the anisotropy of the flow. Smyth and Moum ${ }^{1}$ also consider various spectral measures of the flow, but no examination of the vertical variations within the shear layer is carried out.

A more straight-forward analysis of turbulence anisotropy is found in Thoroddsen and Van Atta, ${ }^{2}$ where the ratios of various components of the Reynolds stress and viscous dissipation rate tensors are reported. Reynolds-stress correlation coefficients, cf., e.g., Kim, Moin, and Moser, ${ }^{3}$ may also shed light on turbulence anisotropy. Lumley ${ }^{4}$ demonstrates the use of the well-known "anisotropy invariant map" in order to characterize the anisotropy of a tensor, whereas Kassinos, Reynolds, and Rogers ${ }^{5}$ show how the structure tensor framework, which comprises non-local information about the flow, can reveal information about anisotropy of the turbulence dimensionality.

Pereira and Rocha ${ }^{6}$ concluded that most second-order closure models inadequately reproduced the anisotropy features of stably stratified homogeneous shear flows. The authors connected this to the structure of the pressure-strain term, which may also be responsible for the inability to predict turbulence flux suppression in such models.

The objectives of the present study are to characterize the dynamic and kinematic responses of an imposed initially stably stratified background on a statistically unsteady shear-generated turbulent flow. Both the smallest dissipative scales as well as the large energy-containing scales will be considered, the latter being particularly important in contaminant transport. The spatial structure of the turbulence is to be investigated by means of the single-point structure dimensionality tensor; the spatial extent of the turbulence structures has direct implications for the deformation of contaminant fields. The analyses are based on data from a Direct Numerical Simulation (DNS) of a temporally evolving Kelvin-Helmholtz (KH) instability at relatively high Reynolds number.

\section{MATHEMATICAL MODELING}

The present analysis is based on data from the DNS of the evolution of KH billows, reported by Werne, Lund, and Fritts. ${ }^{7}$ The Navier-Stokes equations for an incompressible, Newtonian fluid, using the Boussinesq approximation to incorporate effects of density stratification, are given by 


$$
\begin{aligned}
\partial_{i} \tilde{u}_{i} & =0, \\
\partial_{t} \tilde{u}_{i}+\tilde{u}_{j} \partial_{j} \tilde{u}_{i} & =-\frac{\partial_{i} \tilde{p}}{\rho_{0}}+v \partial_{j} \partial_{j} \tilde{u}_{i}+\frac{\rho}{\rho_{0}} g_{i}, \\
\partial_{t} \tilde{\theta}+\tilde{u}_{j} \partial_{j} \tilde{\theta} & =\kappa \partial_{j} \partial_{j} \tilde{\theta},
\end{aligned}
$$

in which $\tilde{u}_{i}=\tilde{u}_{i}(\boldsymbol{x}, t), \tilde{p}=\tilde{p}(\boldsymbol{x}, t)$, and $\tilde{\theta}=\tilde{\theta}(\boldsymbol{x}, t)$ are the instantaneous velocity, pressure, and temperature fields, respectively. Furthermore, $\rho(\boldsymbol{x}, t)$ is the fluid density, $\rho_{0}$ is a reference density, $v$ is the kinematic viscosity, $\kappa$ is the thermal diffusivity, and $g_{i}=(0,0,-g)$ is the gravitational acceleration. Temporal and spatial differentiation is denoted by $\partial_{t}=\partial / \partial t$ and $\partial_{i}=\partial / \partial x_{i}$, respectively, whereas $x_{i}=\left\{x_{1}, x_{2}, x_{3}\right\}$ denotes the streamwise, spanwise, and vertical directions. The Boussinesq approximation entails that $\rho / \rho_{0}=1-\alpha\left(\tilde{\theta}-\Theta_{0}\right)$, where $\alpha$ is the thermal expansion coefficient of the fluid and $\Theta_{0}$ is a reference temperature.

The velocity, pressure, and temperature fields governed by Eqs. (1)-(3) can be decomposed into mean and fluctuating parts, e.g., for the $i$ th component of the velocity field, $\tilde{u}_{i}(\boldsymbol{x}, t)=U_{i}\left(x_{3}, t\right)$ $+u_{i}(\boldsymbol{x}, t)$, where $U_{i}\left(x_{3}, t\right)=\left\langle\tilde{u}_{i}\right\rangle_{x_{1}, x_{2}}$ is the mean velocity and $u_{i}$ is the fluctuating velocity. Here, $\langle\cdot\rangle_{x_{1}, x_{2}}$ denotes the horizontal averaging operator, which approximates the ensemble average, $\langle\cdot\rangle$, in the processing of the DNS data.

The transport equations governing the components of the Reynolds stresses, $\left\langle u_{i} u_{j}\right\rangle$, and turbulent heat flux, $\left\langle u_{i} \theta\right\rangle$, are given by

$$
\begin{aligned}
\partial_{t}\left\langle u_{i} u_{j}\right\rangle+U_{k} \partial_{k}\left\langle u_{i} u_{j}\right\rangle & =\underbrace{-\left(\left\langle u_{j} u_{k}\right\rangle \partial_{k} U_{i}+\left\langle u_{i} u_{k}\right\rangle \partial_{k} U_{j}\right)}_{P_{i j}} \underbrace{-\alpha\left(g_{j}\left\langle u_{i} \theta\right\rangle+g_{i}\left\langle u_{j} \theta\right\rangle\right)}_{G_{i j}} \\
& -\underbrace{2 v\left\langle\partial_{k} u_{i} \partial_{k} u_{j}\right\rangle}_{\varepsilon_{i j}}+v \partial_{k} \partial_{k}\left\langle u_{i} u_{j}\right\rangle-\partial_{k}\left\langle u_{i} u_{j} u_{k}\right\rangle \\
& -\frac{1}{\rho_{0}}\left(\partial_{i}\left\langle u_{j} p\right\rangle+\partial_{j}\left\langle u_{i} p\right\rangle\right)+\underbrace{\frac{1}{\rho_{0}}\left(\left\langle p \partial_{j} u_{i}\right\rangle+\left\langle p \partial_{i} u_{j}\right\rangle\right),}_{\phi_{i j}} \\
\partial_{t}\left\langle u_{i} \theta\right\rangle+U_{k} \partial_{k}\left\langle u_{i} \theta\right\rangle & =-\frac{1}{\rho_{0}}\left\langle\theta \partial_{i} p\right\rangle-\underbrace{-\left(\left\langle u_{k} \theta\right\rangle \partial_{k} U_{i}+\left\langle u_{i} u_{k}\right\rangle \partial_{k} \Theta\right)}_{P_{i \theta}}-\underbrace{(\kappa+v)\left\langle\partial_{k} u_{i} \partial_{k} \theta\right\rangle}_{\varepsilon_{i \theta}} \\
& -\partial_{k}\left(\left\langle u_{i} u_{k} \theta\right\rangle-\kappa\left\langle u_{i} \partial_{k} \theta\right\rangle-v\left\langle\theta \partial_{k} u_{i}\right\rangle\right)-g_{i} \alpha\left\langle\theta^{2}\right\rangle .
\end{aligned}
$$

$P_{i j}$ and $G_{i j}$ denote the rate of production of Reynolds stresses by shear and buoyancy forces, respectively, whereas $\varepsilon_{i j}$ represents the viscous dissipation rate of $\left\langle u_{i} u_{j}\right\rangle . \phi_{i j}$ is the pressure-strain term, which redistributes kinetic energy among the normal components of $\left\langle u_{i} u_{j}\right\rangle . P_{i \theta}$ and $\varepsilon_{i \theta}$ denote the rate of production and rate of viscous dissipation of turbulent heat flux, respectively. Turbulence kinetic energy, its rates of shear and buoyancy production, and its dissipation rate are defined as $k=\frac{1}{2}\left\langle u_{i} u_{i}\right\rangle, P_{k}=\frac{1}{2} P_{i i}, G_{k}=\frac{1}{2} G_{i i}$, and $\varepsilon=\frac{1}{2} \varepsilon_{i i}$, respectively.

The production rates of the Reynolds stresses, temperature fluctuations, and heat fluxes are strongly dependent on each other. Figure 1 illustrates the relations between the most dominating interactions for stratified shear flow and how they are interconnected through their production mechanisms. This will be discussed more thoroughly in Sec. IV C.

As part of the turbulence structure analysis, the so-called structure dimensionality tensor is utilized. The dimensionality tensor is part of the single-point turbulence structure tensor framework described more thoroughly in, e.g., Kassinos, Reynolds, and Rogers ${ }^{5}$ and is defined as

$$
D_{i j}=\left\langle\partial_{i} \Psi_{k} \partial_{j} \Psi_{k}\right\rangle,
$$

in which the fluctuating vector stream function, $\Psi_{i}=\Psi_{i}(\boldsymbol{x}, t), i=1,2,3$, is governed by the Poisson equation

$$
\partial_{k} \partial_{k} \Psi_{i}=-\omega_{i}
$$

Here, $\omega_{i}=\epsilon_{i j k} \partial_{j} u_{k}$ is the fluctuating vorticity field, with $\epsilon_{i j k}$ being the cyclic permutation tensor.

The dimensionality tensor contains information distinctly different from the Reynolds stress tensor; while $\left\langle u_{i} u_{j}\right\rangle$ gives information related to the componental structure of large-scale turbulence, 


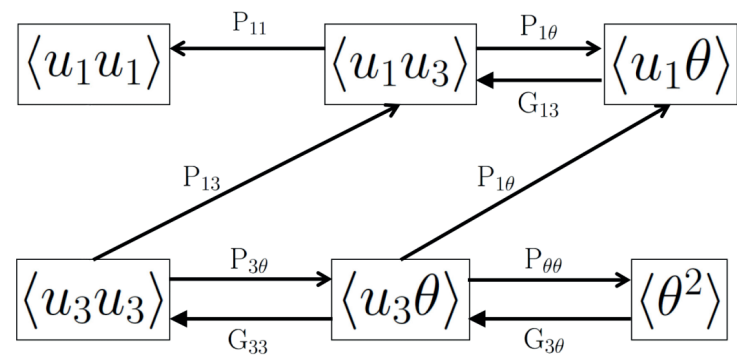

FIG. 1. The dynamically dominant connections between shear and buoyancy production terms in stably stratified shear flow, cf. Eqs. (4) and (5).

$D_{i j}$ carries information about the spatial structure of large-scale turbulence. The Reynolds stresses largely affect how a scalar field is deformed, whereas the dimensionality of the turbulence is more associated with the degree of the deformation. A novelty of the dimensionality tensor is that it indirectly carries non-local structural information about the turbulence through the solution of Eq. (7), despite being a one-point correlation.

In order to characterize large-scale turbulence, the Reynolds stress anisotropy tensor, $b_{i j}$ $=\left\langle u_{i} u_{j}\right\rangle /\left\langle u_{k} u_{k}\right\rangle-\delta_{i j} / 3$, and the dimensionality anisotropy tensor, $d_{i j}=\left\langle\partial_{i} \Psi_{k} \partial_{j} \Psi_{k}\right\rangle /\left\langle\partial_{i} \Psi_{k} \partial_{i} \Psi_{k}\right\rangle$ $-\delta_{i j} / 3$, are used. Small-scale anisotropy will be characterized by the viscous and thermal dissipation rate anisotropy tensors, $e_{i j}=\left\langle\partial_{k} u_{i} \partial_{k} u_{j}\right\rangle /\left\langle\partial_{k} u_{l} \partial_{k} u_{l}\right\rangle-\delta_{i j} / 3$ and $t_{i j}=\left\langle\partial_{i} \theta \partial_{j} \theta\right\rangle /\left\langle\partial_{k} \theta \partial_{k} \theta\right\rangle-\delta_{i j} / 3$.

The second and third invariants of a trace-free, symmetric, second-order tensor can be defined as $I I_{x}=-\frac{1}{2} x_{i j} x_{j i}$ and $I I I_{x}=\frac{1}{3} x_{i j} x_{j k} x_{k i}$, respectively. These are convenient scalar measures of the tensor $x_{i j} . I I_{x}$ measures the degree of anisotropy; $I I_{x}=0$ represents an isotropic state. $I I I_{x}>0$ indicates one dominating tensor component (prolate), whereas $I I I_{x}<0$ implies two dominating components (oblate) (see Figure 2).

It follows from the definitions of the invariants that, for positive semi-definite tensors, $\left(I I_{\mathrm{min}}\right.$, $\left.I I_{\max }\right)=(-1 / 3,0)$ and $\left(I I I_{\min }, I I I_{\max }\right)=(-1 / 108,2 / 27)$. The points $\left(0, I I_{\max }\right),\left(I I I_{\min }, 1 / 12\right)$, and $\left(I I I_{\max },-I I_{\min }\right)$ in the $(I I I,-I I)$ state space define the three vertices in the anisotropy invariant map ${ }^{4}$ shown in Figure 2.

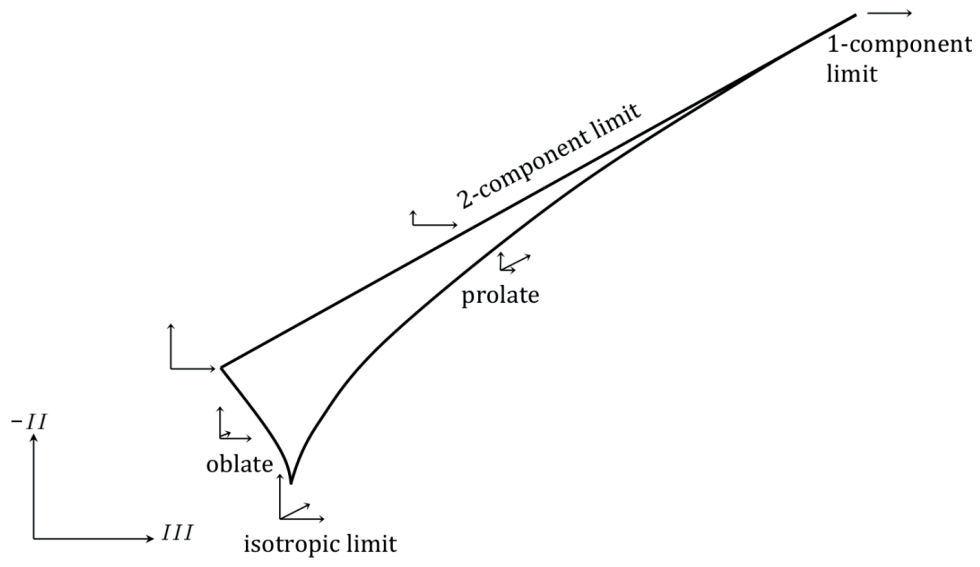

FIG. 2. Illustration of anisotropy invariant map. The three lines represent oblate axisymmetric turbulence, prolate axisymmetric turbulence, and two-componental turbulence, respectively. 
To facilitate the analysis of the different axisymmetric states, the axisymmetry parameter of Lee and Reynolds, ${ }^{8}$ given by

$$
A_{x}=\frac{I I I_{x} / 2}{\left(-I I_{x} / 3\right)^{3 / 2}},
$$

will also be used. This parameter quantifies the degree of turbulence axisymmetry relative to its realizable maximum for a given anisotropy level: $A_{x}=1$ for prolate axisymmetry, whereas $A_{x}=-1$ for oblate axisymmetry.

\section{NUMERICAL MODELING}

Equations (1)-(3) are solved pseudo-spectrally using a streamfunction-vorticity decomposition of the velocity field ${ }^{9}$ that automatically satisfies (1). Derivatives are computed via wavenumber multiplication in spectral space; nonlinear terms are computed via multiplication in physical space. Fast Fourier transforms are used to move between the two spaces. Time stepping is achieved with the low-storage third-order Runge-Kutta method of Spalart, Moser, and Rogers. ${ }^{10}$ The domain size is $4 \lambda \times 2 \lambda \times 2 \lambda$, where $\lambda$ is the wavelength of the most unstable eigenmode of the KH instability. This allows the development of four KH billows, aligned in the streamwise direction, thus enabling far better turbulence statistics than simulations encapsulating only one billow (cf., e.g., Reif et al. ${ }^{11}$ ). The mesh resolution varies as the flow evolves, and the largest grid consists of $3000 \times 1500 \times 1500$ points.

The computational domain is periodic in the two horizontal $\left(x_{1}\right.$ and $\left.x_{2}\right)$ directions. Fixedtemperature, impenetrable, stress-free top and bottom boundaries are used. Initially, at $t^{*}=t U_{0} / H$ $=0$, the flow field consists of small perturbations and the most unstable $\mathrm{KH}$ eigenmode superposed on a background velocity field, $\tilde{\boldsymbol{u}}=\left[U_{0} \tanh \left(x_{3} / H\right), 0,0\right]$. The small perturbations are initiated with a 5/3-Kolmogorov spectrum and a vorticity amplitude of $0.014 U_{0} / H$. The eigenmode initial vorticity amplitude is $0.07 U_{0} / H . H$ and $U_{0}$ denote half the initial shear-layer depth and the free-stream velocity, respectively. The initial temperature field is $\tilde{\theta}=\beta x_{3}$, where $\beta$ is a constant.

The parameters characterizing the initial flow fields are the Richardson number, $R i$ $=g \alpha \beta H^{2} / U_{0}^{2}=0.05$, the Reynolds number, $R e=U_{0} H / v=2500$, and the Prandtl number, $\mathrm{Pr}$ $=v / \kappa=1$. Further details concerning the simulation can be found in Werne, Lund, and Fritts. ${ }^{7}$

The temporal evolution of KH billows is discussed in, e.g., Werne, Lund, and Fritts, ${ }^{7}$ Fritts et al..${ }^{12}$ Palmer, Fritts, and Andreassen, ${ }^{13}$ Thorpe, ${ }^{14}$ and Smyth and Moum,,${ }^{15}$ and it can be summarized briefly as follows: Initial temperature (or, equivalently, density) perturbations in combination with a mean vertical gradient of streamwise velocity lead to KH waves. The amplitude of these waves grows, and eventually, the waves break (see Figure 3, top). The flow subsequently becomes fully turbulent. Turbulent motion is generated by means of velocity shear caused by convective

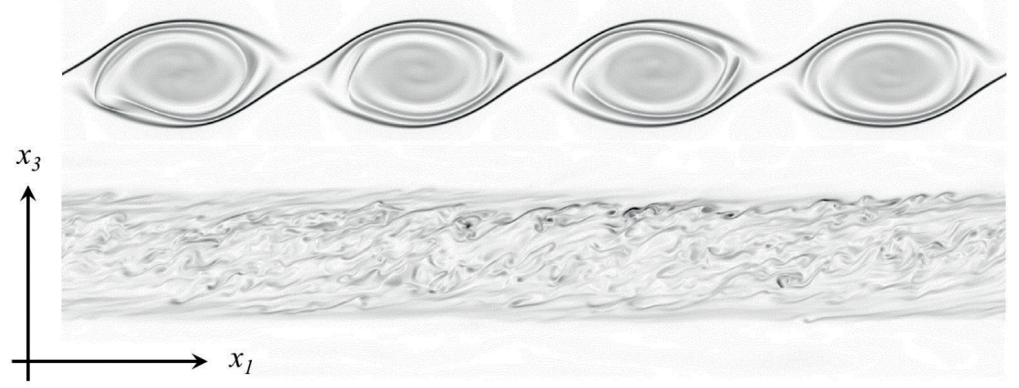

FIG. 3. Contours of instantaneous vorticity magnitudes at $t^{*}=50$ (top) and $t^{*}=227$ (bottom). It should be noted that $x_{3}=0$ corresponds to the center of the shear layer. Cf. also Figure 4. 


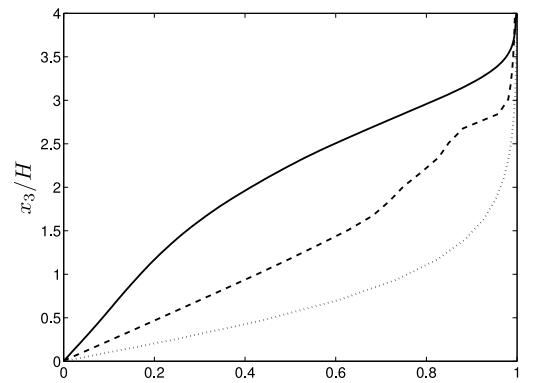

(a) $U_{1}^{*}=U_{1} / U_{0}$

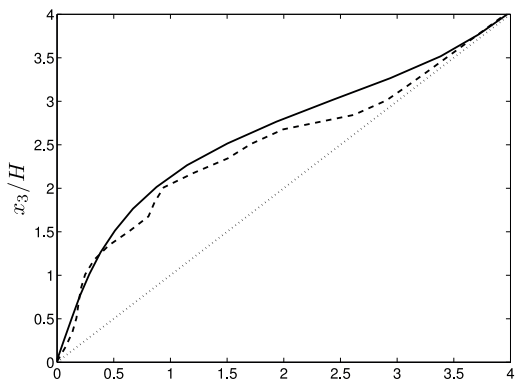

(b) $\Theta^{*}=\Theta /(\beta H)$

FIG. 4. Non-dimensional mean velocity and mean temperature profiles, across half the shear layer, at $t^{*}=0(\cdots \cdots), 50$ (---), and 227 (-). Cf. also Figure 3.

instabilities and wave breaking. As the flow field evolves, it becomes nearly horizontally homogeneous (see Figure 3, bottom). The effect of the stably stratified background becomes increasingly dominant, suppressing the turbulence intensity and eventually causing relaminarization. Weak gravity waves are also present, but this phenomenon is not investigated presently. Figure 4 shows the initial mean profiles of velocity and temperature, as well as the profiles for the two time instances shown in Figure 3.

\section{RESULTS AND DISCUSSION}

The present study addresses the net kinetic energy decay phase of the statistically unsteady KH evolution, cf. Figure 5. During this phase, stratification increases and the turbulence becomes horizontally homogeneous. The turbulence statistics can therefore be obtained by averaging in the horizontal directions. In addition, all quantities symmetric about the vertical centerline, $x_{3}=0$, are also averaged by reflection about the symmetry axis to improve statistics.

Figure 5 shows the change in time of turbulence kinetic energy integrated across the shear layer, $k^{*}=\int k \mathrm{~d} x_{3} /\left(U_{0}^{2} H\right)$. In the following, three selected instances in time will be in focus (cf. Figure 5), which roughly correspond to the early, middle, and late stages of the net energy decay

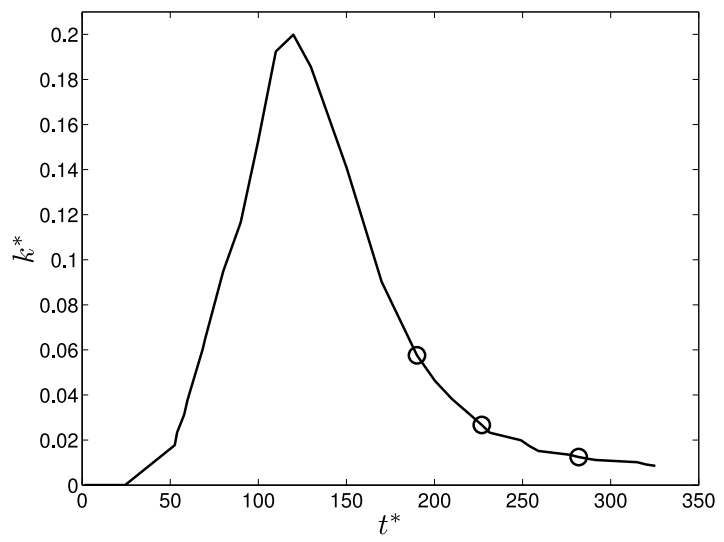

FIG. 5. Change of integrated turbulence kinetic energy, $k^{*}=\int k \mathrm{~d} x_{3} /\left(U_{0}^{2} H\right)$, in time. The symbols mark $t^{*}=190,227$, and 282. The net kinetic energy decay phase implies $t^{*} \gtrsim 120$. 


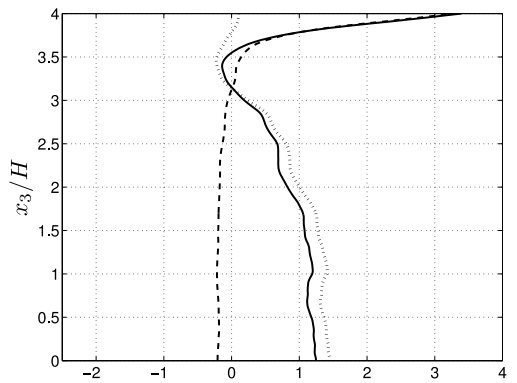

(a) $t^{*}=190$

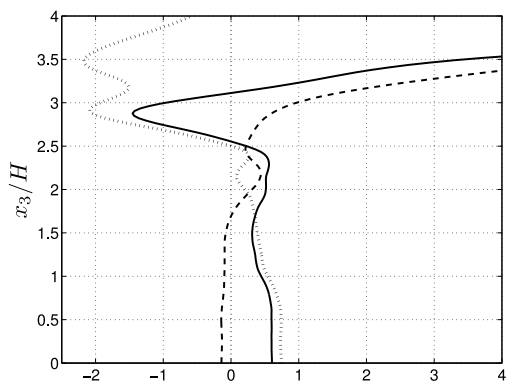

(b) $t^{*}=282$

FIG. 6. Ratio of turbulent production to turbulent dissipation rate across half the shear layer. $P_{k} / \varepsilon(\cdots \cdots), G_{k} / \varepsilon(---)$, and $\left(P_{k}+G_{k}\right) / \varepsilon(-)$.

phase. Even though there is a net decay of turbulence kinetic energy at $t^{*}=190$, a portion of the domain exhibits positive production of turbulence kinetic energy, as shown in Figure 6(a).

Note that for the late decay stage, it is the negative rate of shear production that causes $\left(P_{k}+G_{k}\right) / \varepsilon<0$, contrary to the more common case where positive shear production is countered by buoyancy. During this phase, kinetic energy is transferred from the turbulence to the mean flow.

Figure 7 shows the vertical variation of the gradient Richardson number, $\mathrm{Ri}_{g}$, across half the shear layer. This dimensionless number represents the ratio of buoyancy to shear forcing and is here given by

$$
\mathrm{Ri}_{g}=\frac{\alpha g \partial_{3} \Theta}{\left(\partial_{3} U_{1}\right)^{2}} .
$$

It has been shown, both theoretically ${ }^{16}$ and experimentally, ${ }^{17}$ that flows with a gradient Richardson number less than the critical value of $\mathrm{Ri}_{g, c}=0.25$ are dynamically unstable. Gradient Richardson numbers above this value tend to imply locally stable flow, in which the turbulence is significantly affected by buoyancy. However, due to the spatially and temporally varying nature of stratified turbulence, large instantaneous - or even mean — values of the gradient Richardson number at a given position are by no means a sufficient sign of laminar flow, as long as the flow exhibits lower Richardson numbers at other positions.

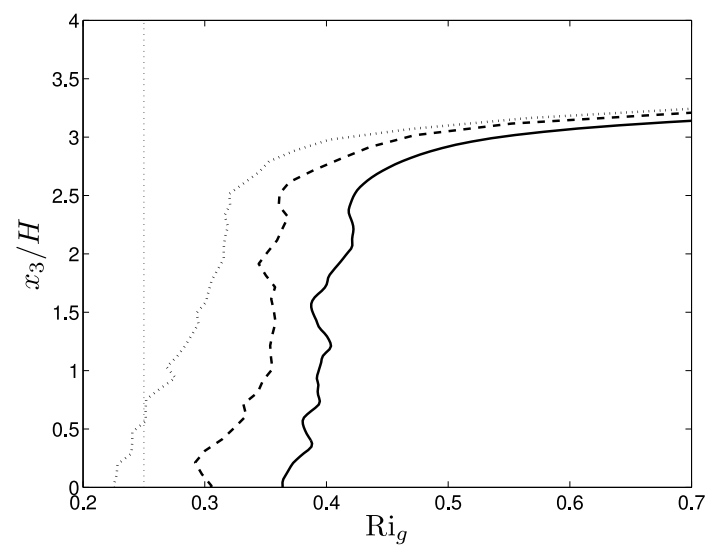

FIG. 7. Variation of the gradient Richardson number across half the shear layer at $t^{*}=190(\cdots \cdots), t^{*}=227(---)$, and $t^{*}=282(-)$. The critical Richardson number, $\mathrm{Ri}_{g, c}=0.25$, is indicated by a vertical line. 
It can be observed in Figure 7 that the gradient Richardson number increases with time across the entire shear layer and that maximum stratification occurs close to the edge of the shear layer $\left(x_{3} \approx 3.5 H\right)$. In fact, as the mean velocity gradient tends toward zero with $\partial_{3} \Theta \neq 0, \mathrm{Ri}_{\mathrm{g}} \rightarrow \infty$. Hence, buoyancy completely dominates the flow in this region. Except for the earliest time considered, $\mathrm{Ri}_{g}>\mathrm{Ri}_{g, c}$ across the entire layer.

\section{A. Anisotropy}

In laboratory experiments of decaying stratified turbulence, Thoroddsen and Van Atta $^{2}$ noted that small-scale anisotropy seemed to develop faster than large-scale anisotropy. This is unexpected in light of the common supposition that anisotropy related to stratification is imposed directly only on the larger scales and that small-scale turbulence only indirectly is affected through the cascade process. ${ }^{18,19}$

The observation of Thoroddsen and Van Atta ${ }^{2}$ was made in the absence of mean shear, whereas in KH flow mean shear initially dominates and remains important throughout the entire evolution. Figure 8 shows the second invariants of the anisotropy tensors of Reynolds stress $\left(I I_{b}\right)$, viscous dissipation rate $\left(I I_{e}\right)$, thermal dissipation rate $\left(I I_{t}\right)$, and dimensionality $\left(I I_{d}\right)$ at different times. It can be observed that the relatively faster increase in viscous dissipation rate anisotropy also occurs in sheared stratified flow. Furthermore, it can be observed that the increase in anisotropy is larger near the edge of the shear layer, where the gradient Richardson number is higher. At the layer edge, where the stratification locally is strong, the dissipation rate anisotropy actually surpasses the Reynolds stress anisotropy. For $\mathrm{Ri}_{g} \lesssim \mathrm{Ri}_{g, c}$, the rate of viscous dissipation is almost isotropic.

Componental structure information is carried by both the second invariants of the Reynolds stress anisotropy tensor $\left(I I_{b}\right)$ and the viscous dissipation rate anisotropy tensor $\left(I I_{e}\right)$. In the center of

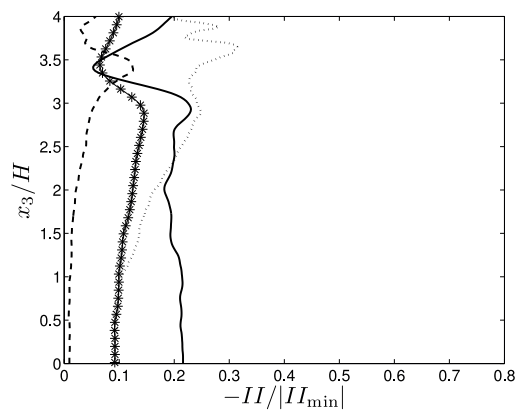

(a) $t^{*}=190$

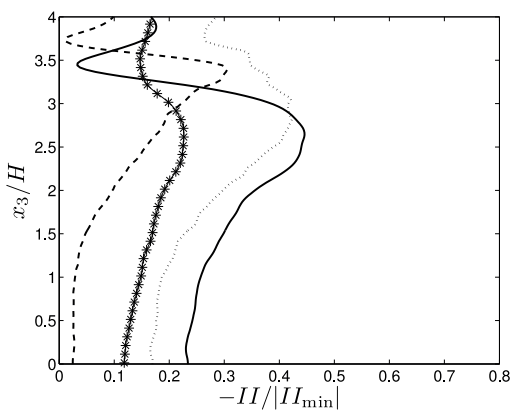

(b) $t^{*}=227$

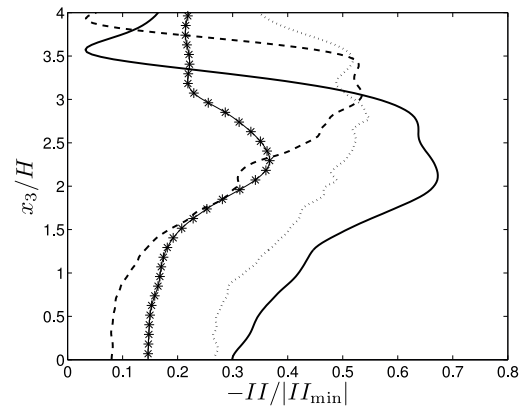

(c) $t^{*}=282$

FIG. 8. Second invariants of the Reynolds stress (-), viscous dissipation rate (---), thermal dissipation rate ( $\cdots \cdots)$, and dimensionality (—) across half the shear layer. $\left|I I_{\min }\right|=1 / 3$. 
the layer, both large- and small-scale anisotropy increase with time. Even if the level of large-scale componental anisotropy always exceeds that of the smaller scales, the small-scale componental anisotropy has a larger percentage increase with time.

As seen in Figure 8(a), the second invariant of the thermal dissipation anisotropy tensor $\left(I I_{t}\right)$ differs greatly from $I I_{e}$ even though they both represent small scale anisotropy. $I I_{t}$ is much more anisotropic. As time evolves, it is evident that the anisotropy of viscous dissipation becomes more similar to the anisotropy of the thermal dissipation in the edge region, whereas the difference in the center remains approximately the same. Generally, these results suggest that buoyancy has a stronger impact on local anisotropy than does mean shear in the present flow configuration. This is in accordance with the theoretical results of Reif and Andreassen, ${ }^{20}$ which suggest that only for very small gradient Richardson numbers $\left(\mathrm{Ri}_{g} \ll 1\right)$ can buoyancy effects on local isotropy be ignored in the presence of mean shear.

Figure 8 also reveals an apparent paradox, which is most prominent for the larger scales: As stratification increases in time, the anisotropy clearly increases across the shear layer. In the region $x_{3} \lesssim 2.5 H$, this is consistent with a similar trend of increasing anisotropy with coincident increasing stratification as one moves closer to the edge region. However, close to the layer edge $\left(x_{3} \gtrsim 2.5 H\right)$, a reversal of this relationship is evident; increased stratification (cf. Figure 7) is accompanied by a sudden decrease in anisotropy! This pattern holds for every time instance, albeit with a slight variability in the vertical position at which the anisotropy begins to subside.

The results also suggest that in the net decay phase, the location of maximum Reynolds stress anisotropy consistently occurs at $0.3<\mathrm{Ri}_{g}<0.45$, as shown in Figure 9 . For late times $\left(t^{*} \gtrsim 250\right)$, the values are close to 0.4 . That is, up to a certain level of stratification $\left(\mathrm{Ri}_{g} \approx 0.4\right)$ anisotropy increases, but for stronger levels of stable stratification, anisotropy begins to decrease.

Figures 5 and 6 show that the turbulence kinetic energy decreases rapidly and that the turbulent dissipation rate significantly exceeds the turbulent production rate when stratification increases. This indicates relaminarization. Since the anisotropy primarily is caused by the rate of production term, absence of this mechanism is associated with "return to isotropy." 21 Hence, the combination of reduced net production and rapidly decreasing turbulence kinetic energy levels indicates that relaminarization effects are responsible for the reduced anisotropy as $\mathrm{Ri}_{g} \gtrsim 0.4$.

Interestingly, in the case of wall-bounded stratified channel flow, Garcia-Villalba and Del Alamo $^{22}$ display a similar trend related to density fluctuations; as the stratification level increases, the normalized root-mean-square density fluctuations increase up to a certain level. However, for very strong levels of stratification, the fluctuations suddenly decrease again. The corresponding value of the critical gradient Richardson number agrees rather well with that found presently. In

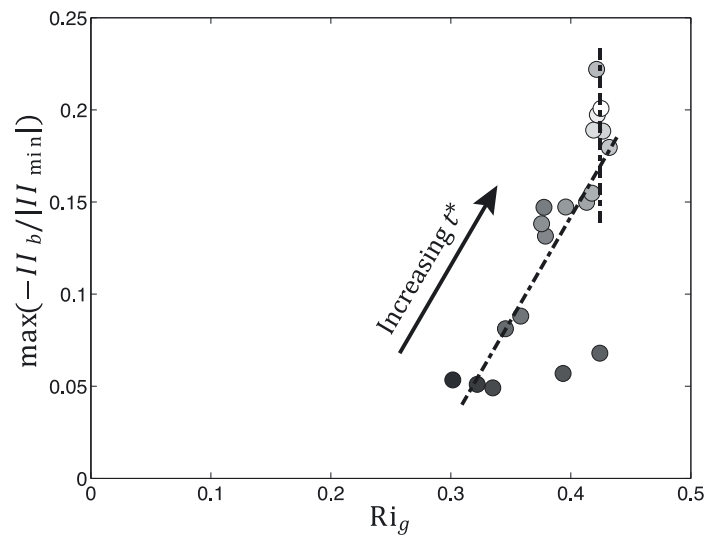

FIG. 9. Maximum values of the second invariant of the Reynolds stress anisotropy tensor within the shear layer $\left(x_{3} \leq 3.5 H\right)$ vs. the corresponding gradient Richardson number. Circles represent different times in the net kinetic energy decay phase, with lighter color implying later times. 
their paper, Garcia-Villalba and Del Alamo ${ }^{22}$ use internal gravity waves as an explanation for altered density fluctuations in the channel core region, but it is unclear whether they mean the increased fluctuations in general or the particular sudden decrease at higher stratification levels. If it is the latter, an explanation is lacking of how the presence of stronger internal gravity waves can reduce the density fluctuation levels. In the present case, gravity waves are indeed dominant in the layer edge, but such waves (quantified by, e.g., the Brunt-Väisälä frequency) are significantly less correlated with $I I_{b}$ and $\mathrm{Ri}_{g}$.

The second invariant of the dimensionality tensor, $I I_{d}$, expresses the deviation from spherically coherent flow structures (in an average sense). It is seen, from Figure 8, that the magnitude of $I I_{d}$ remains almost constant in time and space, except for the final time instance, where a notable peak appears near the edge region. This peak coincides with the peak in $I I_{b}$.

Another interesting feature of the anisotropy regards the turbulence axisymmetry, the state of which can be inferred from the axisymmetry parameter (Figure 10). For the Reynolds stress anisotropy tensor, $A_{b}=1$ indicates a one-componental (1C) axisymmetric state and $A_{b}=-1$ indicates a two-componental (2C) axisymmetric state. Aside from the physical implications related to the componentality of the flow, the state of axisymmetry may be an issue in turbulence modeling.

The edge layer seems to be characterized by a $1 \mathrm{C}$ state, except for one narrow band in the immediate vicinity of the edge $\left(x_{3} \approx 3.5 H\right)$, where the Reynolds stresses tend towards an increasingly more $2 \mathrm{C}$ state. Inspection of the Reynolds stress components at the latter location reveals that $\left\langle u_{3}^{2}\right\rangle>\left\langle u_{1}^{2}\right\rangle$, meaning that the dominant Reynolds stresses lie in the $x_{2}-x_{3}$ plane. This may indicate increased relative vertical motion near the edge as the stratification level increases. However, the Reynolds stresses are 1C in the center region, most likely associated with jet-like motion. Here, $\left\langle u_{3}^{2}\right\rangle /\left\langle u_{1}^{2}\right\rangle$ decreases with time and thus also with increased stratification levels. In fact, by looking

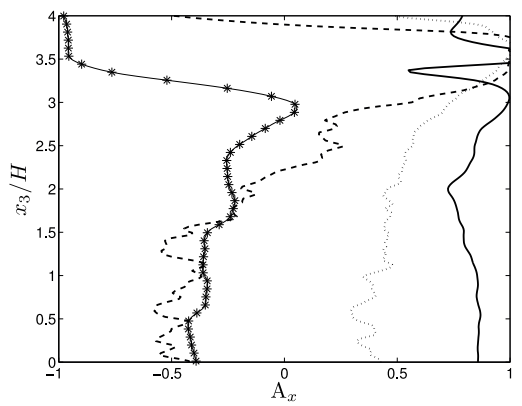

(a) $t^{*}=190$

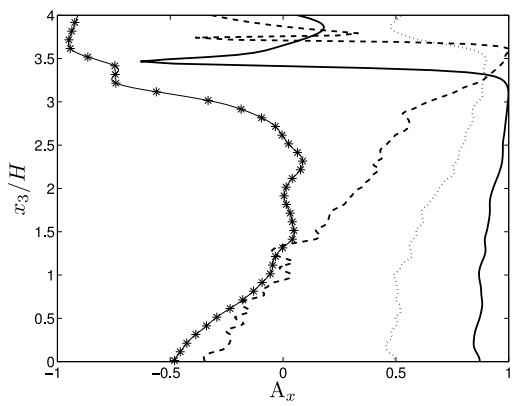

(b) $t^{*}=227$

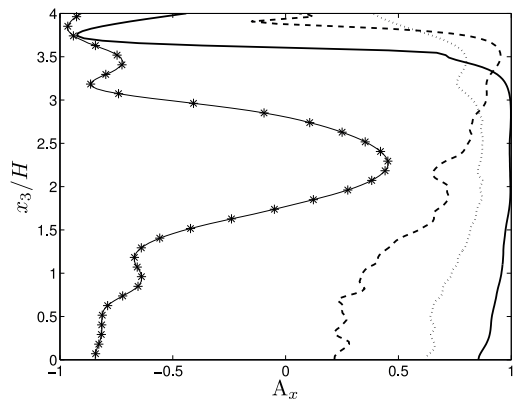

(c) $t^{*}=282$

FIG. 10. Axisymmetry parameter values for Reynolds stress (-), viscous dissipation rate (---), thermal dissipation rate $(\cdots \cdots)$, and dimensionality $(\multimap)$ across half the shear layer. 
at the values of $\left\langle u_{3}^{2}\right\rangle /\left\langle u_{1}^{2}\right\rangle$ against $\mathrm{Ri}_{g}$ for all instances in time, a similar critical point is found as for the second invariants (cf. Figure 9); up to $\mathrm{Ri}_{g} \approx 0.4$, relative vertical motion decreases, whereas for larger values of the gradient Richardson number, relative vertical motion increases.

For the other anisotropy tensors $\left(e_{i j}, t_{i j}\right.$, and $\left.d_{i j}\right)$, the value of the axisymmetry parameter have a different physical interpretation. $A_{x}=1$ implies two-dimensional (2D) structures, whereas $A_{x}=-1$ implies one-dimensional (1D) structures. The dissipative velocity scales change from 1D to $2 \mathrm{D}$ in the center region as stratification increases. Near the edge, the small scales are always 2D, reminiscent of near-wall structures. The dissipative thermal scales are 2D in the entire shear layer, but more so near the edge, meaning that the vertical variations of temperature fluctuations are much more pronounced than the horizontal variations.

The $2 \mathrm{D}$ axisymmetry levels of the dimensionality are also seen to peak around $x_{3} \approx 2.5 \mathrm{H}$ for $t^{*}=282$, cf. Figure 10(c). At earlier times, there is a low degree of axisymmetry (i.e., $\left|A_{d}\right| \ll 1$ ) at this location. Closer to the centerline and at the edge, the dimensionality is generally highly $1 \mathrm{D}$ for all times. However, this does not necessarily imply that the structures at these locations are equal in every respect; on the contrary, the centerline structures are relatively shorter in the streamwise direction than the structures near the edge, which are more likely to resemble the coherence of near-wall streaks. In combination with information about the individual dimensionality tensor components, cf. Figure 11, these results reveal structural information about the flow.

In the center region, the coherence of the Reynolds stresses is organized in ellipsoids which are stretched in the streamwise direction. Initially, these structures are relatively circular in the $x_{2}-x_{3}$ plane, but eventually they become more compressed in the vertical direction. Closer to the edge $\left(1.5 H \lesssim x_{3} \lesssim 2.5 H\right)$, the ellipsoids are much "flatter" for all times, and this feature is also exaggerated with time. At the edge $\left(x_{3} \approx 3.5 H\right)$, however, the data imply 1D structures. This information is included in Figure 11 as conceptual illustrations of the spatial extent of the turbulence structures for three different vertical locations at $t^{*}=282$. The turbulence structures are approximately parallel $\left(0^{\circ}-20^{\circ}\right.$ tilting $)$ to the $x_{1}$-axis for all times and positions.

\section{B. Temporal scales}

The fluctuating velocity and temperature fields can both be associated with specific length and time scales. For the velocity field, the turbulent time scale is given by $T=k / \varepsilon$, whereas for the temperature fluctuations, an appropriate time scale is $T_{\theta}=\left\langle\theta^{2}\right\rangle / \varepsilon_{\theta}$. Here, $\varepsilon_{\theta}=2 \kappa\left\langle\partial_{i} \theta \partial_{i} \theta\right\rangle$ is the thermal dissipation rate. These scales represent the larger scales of turbulent motions.

For the turbulent velocity field, the smallest time scale, i.e., the Kolmogorov time scale, is given by $\tau=\sqrt{v / \varepsilon}$. However, to the knowledge of the authors, no equivalent smallest time scale

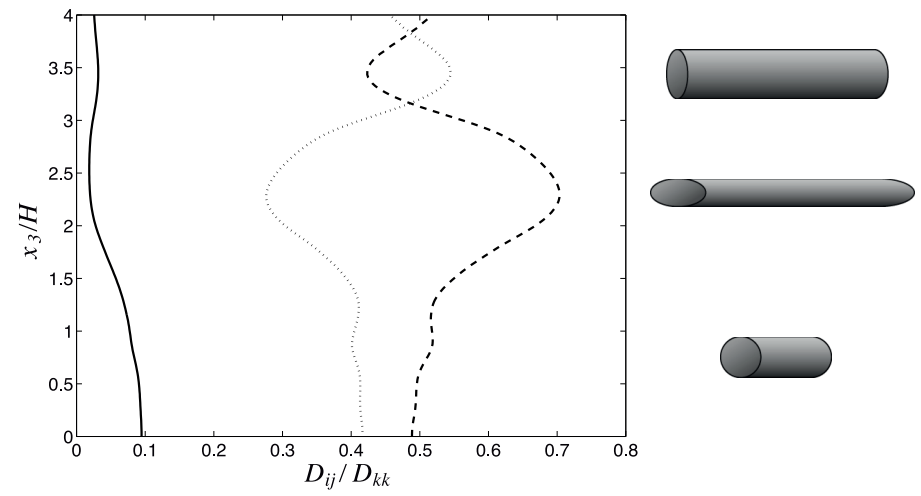

FIG. 11. Normal components of the dimensionality tensor across half the shear layer at $t^{*}=282 . D_{11} / D_{k k}(-), D_{22} / D_{k k}$ $(\cdots \cdots)$, and $D_{33} / D_{k k}(---)$. Illustrations of the spatial extent of the turbulence structures are included at three vertical locations. 


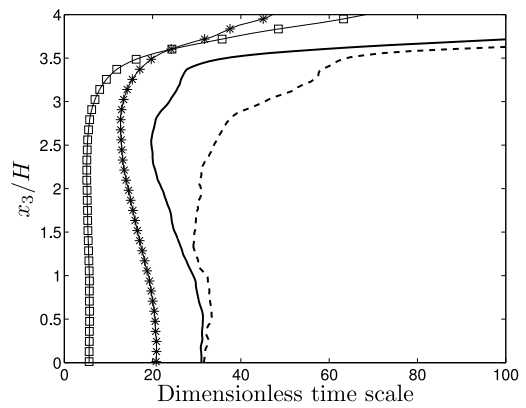

(a) $t^{*}=190$

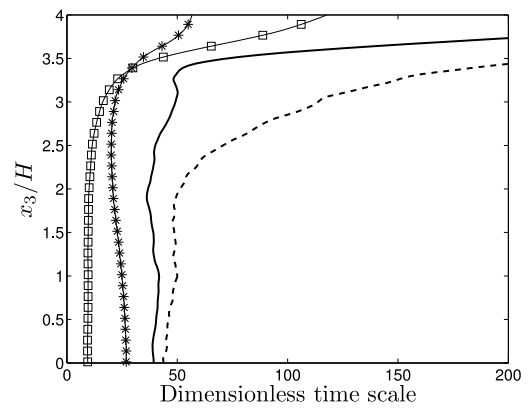

(b) $t^{*}=227$

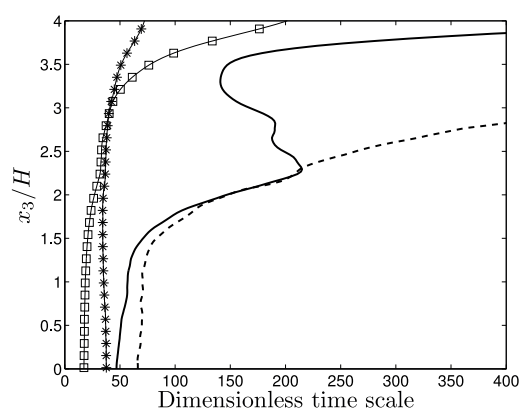

(c) $t^{*}=282$

FIG. 12. Time scales for velocity and temperature. $T=k / \varepsilon(-), T_{\theta}=\left\langle\theta^{2}\right\rangle / \varepsilon_{\theta}(---), 5 \tau=5 \sqrt{v / \varepsilon}(-\square)$, and $5 \tau_{\theta}=$ $5 \sqrt{\kappa \varepsilon_{\theta} /\left(\varepsilon_{i \theta} \varepsilon_{i \theta}\right)}(\multimap-$.

for the temperature field have been defined. The commonly used Batchelor scale is unsuitable, as it implicitly assumes $\varepsilon_{\theta} \sim \varepsilon$ and is relevant mainly for $\operatorname{Pr} \gg 1$ (Wyngaard ${ }^{23}$ ). The authors therefore propose to use a thermal time scale given by $\tau_{\theta}=\kappa^{1 / 4} /\left(\varepsilon_{\theta} g^{2} \alpha^{2}\right)^{1 / 4}$.

The temporal scales associated with the fluctuating velocity and temperature fields are shown in Figure 12. At the edge of the layer, the difference between the two large scales generally increases with increased stratification, the thermal scale being the largest. In the inner region of the shear layer, on the other hand, the ratio between the scales is closer to unity for all times, with relatively small variations in the vertical direction. Stratification appears to affect the large thermal scale to a higher degree than it affects the large turbulence scale.

The small scales become increasingly similar with time. The thermal scale is slightly larger in the center of the layer, but this situation is reversed at the outer edge, where stratification is larger. It thus seems that for a certain level of stratification, the small velocity scale will surpass the small thermal scale. There is generally little vertical variation in the small scales across the layer, in comparison to the large scales.

The scale separation between large and small velocity scales evolves differently in the center region than at the layer edge; in the former case, scale separation decreases with time, consistent with the monotonic reduction of the Reynolds number. At the edge of the layer, however, the scale separation generally increases at later time instances, due to the increase of the large time scale at $x_{3} \approx 2.5 \mathrm{H}$. In contrast, the thermal scale separation remains $O(10)$ in the center region for all times considered, but the separation increases in the layer edge, where the large scale increases significantly. These results corroborate the notion that stratification affects larger scales to a higher degree than it affects smaller scales.

Figure 13 shows the variation of the ratio of mechanical to thermal time scales, $\mathcal{R}=T / T_{\theta}$, across the shear layer. This ratio is commonly used to device a simple model for $\varepsilon_{\theta}$, and in the 


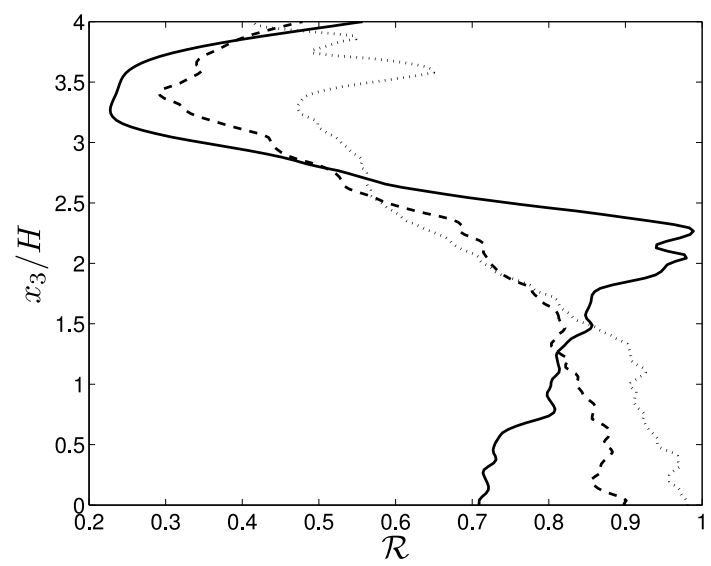

FIG. 13. Ratio of mechanical to thermal time scale, $\mathcal{R}=(k / \varepsilon)\left(\left\langle\theta^{2}\right\rangle / \varepsilon_{\theta}\right)^{-1}$, across half the shear layer at $t^{*}=190(\cdots \cdots)$, $t^{*}=227(---)$, and $t^{*}=282(-)$.

presence of a uniform mean temperature gradient without mean shear, it is found to be a constant equal to approximately 1.5 (Sirivat and Warhaft ${ }^{24}$ ). As seen in Figure 13, the ratio seems to have a fairly constant value of $\mathcal{R} \approx 0.85$ in the inner, shear-dominated region, but as stratification increases, the ratio significantly decreases at the edge of the layer. The imposition of stratification thus seems to affect the velocity and temperature fields differently. For decaying temperature fluctuations in grid generated turbulence, $\mathcal{R}=m / n$, where $m$ and $n$ are the exponents of the power-law decays for $\left\langle\theta^{2}\right\rangle$ and $k$, respectively. Even though the value of the mechanical to thermal time scale ratio found here differs from that found by Sirivat and Warhaft, ${ }^{24}$ the relation between $m$ and $n$ still gives a good approximation of $\mathcal{R}$ despite the presence of shear. Improved models for the thermal dissipation might take stratification into account directly, cf., e.g., Weinstock ${ }^{25}$ and Fossum, Wingstedt, and Reif. $^{26}$

\section{Layer-edge physics}

The physics in the strongly vertically inhomogeneous shear-layer edge differs significantly from the inner region which remains almost homogeneous. The observation of large anisotropies, even on smaller scales, poses significant challenges for turbulence models. As an example, the most common dissipation rate models of today assume local isotropy. Figure 14 shows the ratio of turbulence to mean flow time scale, which should be $S k / \varepsilon \ll 1$ for the local isotropy assumption to hold. ${ }^{20}$ Clearly, this is not satisfied in the current simulation. From considerations in Durbin and Reif, ${ }^{27}$ pp. $170-171$, it can be shown that for homogeneously sheared turbulence in equilibrium, $S k / \varepsilon \approx 5$, which is also the condition for which most commonly used Reynolds-Averaged Navier-Stokes turbulence models are calibrated. However, from Figure 14, it is seen that when stratification dominates, the value of $S k / \varepsilon$ becomes much larger. $S k / \varepsilon \gg 1$ implies that the turbulence time scale is much larger than the mean flow time scale, indicating a laminarization process which might be difficult to capture with contemporary turbulence models.

Figure 15 shows the variation of the Reynolds stress components across the shear layer. Contrary to near-wall behavior (cf., e.g., Moser, Kim, and Mansour ${ }^{28}$ ), the figure shows that as the edge of the shear layer is approached, $\left\langle u_{3}^{2}\right\rangle /\left\langle u_{1}^{2}\right\rangle \rightarrow 0$. Furthermore, it can be observed that momentum flux reversal occurs in the edge layer, i.e., that $\left\langle u_{1} u_{3}\right\rangle$ changes sign. This phenomenon becomes more pronounced as the stratification increases, and it has several implications that will be discussed below.

In order to provide an explanation of the flux reversal, a first step is to look at the rate of production of $\left\langle u_{1} u_{3}\right\rangle$, i.e., $\mathcal{P}_{13}=P_{13}+G_{13}=-\left\langle u_{3}^{2}\right\rangle \partial_{3} U_{1}+\alpha g\left\langle u_{1} \theta\right\rangle$. From Figures 15 and 16 , it is 


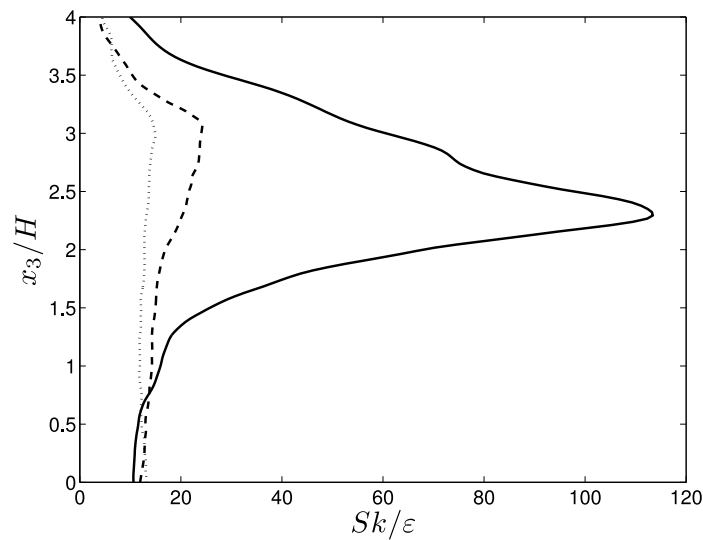

FIG. 14. Turbulence to mean flow time scale ratio, $S k / \varepsilon$, across half the shear layer at $t^{*}=190(\cdots \cdots), t^{*}=227(---)$, and $t^{*}=282(-)$.

evident that whereas $\left\langle u_{3}^{2}\right\rangle / 2 k$ generally increases near the edge, $\left\langle u_{1} \theta\right\rangle / \sqrt{\left\langle u_{i} \theta\right\rangle\left\langle u_{i} \theta\right\rangle}$ decreases and eventually even changes sign. From an inspection of $\mathcal{P}_{13}$ (figure not shown here), it is seen that total production of $\left\langle u_{1} u_{3}\right\rangle$ becomes negative near the layer edge. Unless the shear component is supplied with energy via the pressure-strain term, it would rapidly change sign and thus eventually cause relaminarization.

A consequence of the momentum flux reversal concerns the turbulence kinetic energy. It can be found that the total production of turbulence kinetic energy, i.e., the sum of both shear and buoyancy production terms, $P_{k}$ and $G_{k}$, respectively, reduces to $\mathcal{P}_{k}=P_{k}+G_{k}=\frac{1}{2} P_{11}+\frac{1}{2} G_{33}$ $=-\left\langle u_{1} u_{3}\right\rangle \partial_{3} U_{1}+\alpha g\left\langle u_{3} \theta\right\rangle$ for the present case. Hence, it can be seen that reversal of $\left\langle u_{1} u_{3}\right\rangle$ leads to a change of role of the shear production part of $\mathcal{P}_{k}$. Normally, both in neutral flows and as seen presently in the layer center $\left(x_{3} \lesssim 2 H\right)$ in Figure 6 , shear produces turbulence kinetic energy, whereas buoyancy acts to decrease the energy. At the edge, however, shear actually seems to remove energy from the fluctuating field. Similarly, the buoyancy part of the production of turbulence kinetic energy, $G_{k}$, also changes sign near the layer edge, as a consequence of heat flux reversal

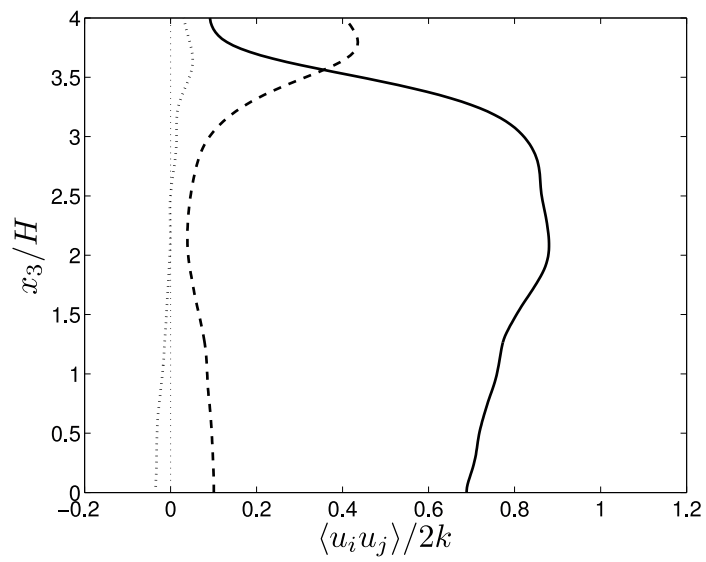

FIG. 15. Normalized Reynolds stress components across half the shear layer for $t^{*}=282 .\left\langle u_{1}^{2}\right\rangle / 2 k(-),\left\langle u_{3}^{2}\right\rangle / 2 k(---)$, and $\left\langle u_{1} u_{3}\right\rangle / 2 k(\cdots \cdots)$. The zero Reynolds stress level is marked by a vertical line. 


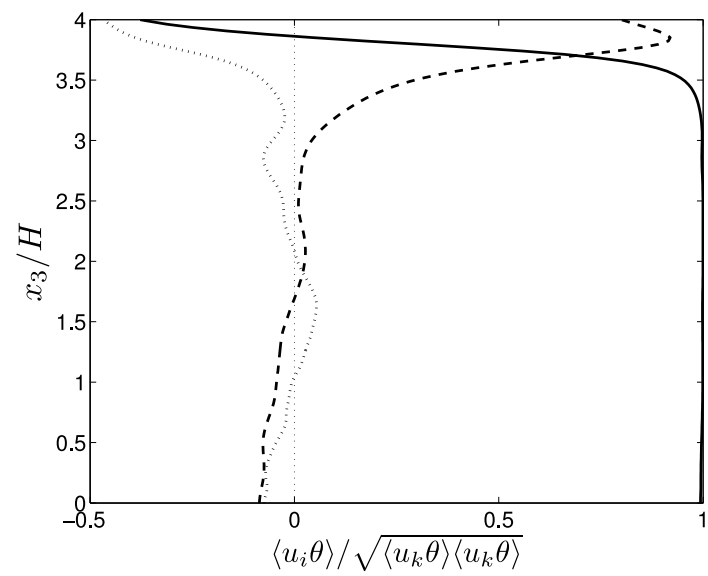

FIG. 16. Heat flux components across half the shear layer for $t^{*}=282$. $\left\langle u_{1} \theta\right\rangle / \sqrt{\left\langle u_{k} \theta\right\rangle\left\langle u_{k} \theta\right\rangle}(-),\left\langle u_{2} \theta\right\rangle / \sqrt{\left\langle u_{k} \theta\right\rangle\left\langle u_{k} \theta\right\rangle}$ $(\cdots \cdots)$, and $\left\langle u_{3} \theta\right\rangle / \sqrt{\left\langle u_{k} \theta\right\rangle\left\langle u_{k} \theta\right\rangle}(---)$. The zero heat flux level is marked by a vertical line.

(i.e., the sign change of $\left\langle u_{3} \theta\right\rangle$ ), cf. Figure 16. In conclusion, the shear and buoyancy contributions to the turbulence kinetic energy production change roles near the edge of the KH layer.

The evolution of turbulence production can be analyzed, for instance, by looking at the ratio of total turbulence kinetic energy production to turbulence dissipation rate (i.e., $\mathcal{P}_{k} / \mathcal{E}$ ) at different times (see Figure 6). This ratio is found to be close to or below zero and decreasing with time near the layer edge, suggesting a permanent decay of turbulence, which increases with increased stratification. In the layer center, $\mathcal{P}_{k} / \varepsilon<1$ at later times, implying turbulence decay locally in this region. In the outer edge, however, the production-dissipation ratio is much larger than unity, solely due to buoyant production, owing to large vertical heat flux, $\left\langle u_{3} \theta\right\rangle$.

Finally, some attention should be given to the pressure-strain tensor, as this represents the crucially important mechanism by which energy is redistributed among the normal components of the turbulent stress tensor. A straight-forward inspection of Figure 17 shows that for parts of the domain, the sign of $\mathcal{P}_{11}=P_{11}+G_{11}=P_{11}$ is the same as the sign of the pressure-strain term, $\phi_{11}$. This is irregular and not captured by common pressure-strain models, such as the simple

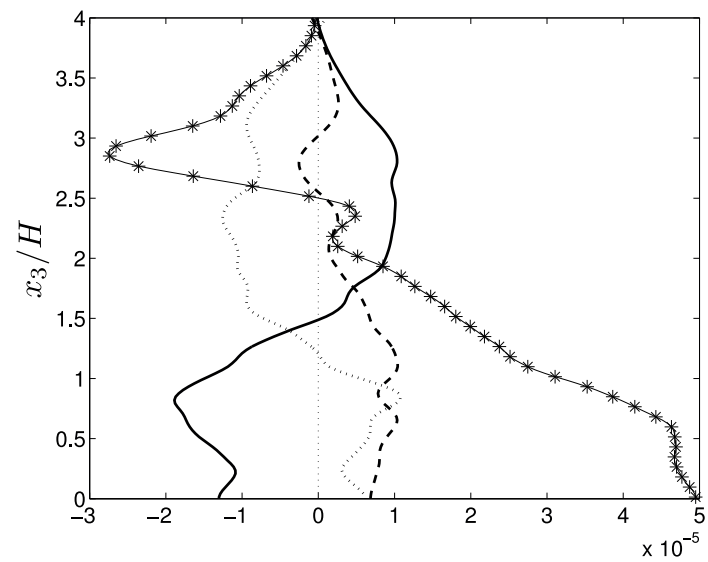

FIG. 17. Pressure-strain components and total production of streamwise Reynolds stresses across half the shear layer for $t^{*}=282 . \phi_{11}(-), \phi_{22}(---), \phi_{33}(\cdots \cdots)$, and $P_{11}+G_{11}(-*)$. 
isotropization of production (IP) model. ${ }^{29}$ It is interesting to note that in the region where $\left\langle u_{1}^{2}\right\rangle$ is largest, around $x_{3} \approx 1.5 H-2 H$ (cf. Fig. 15), the pressure-strain correlation acts as a source term together with $\mathcal{P}_{11}$.

The vertical pressure-strain component, $\phi_{33}$, changes sign for $x_{3} \gtrsim 1.5 H$, contrary to common free shear flow behavior. This is similar to what is observed in the near-wall region of wall-bounded flows ${ }^{28}$ corroborating the notion that strong stable stratification may in some ways affect turbulence like an impermeable wall.

\section{CONCLUDING REMARKS}

DNS data of an evolving KH instability have been analyzed, with particular emphasis on anisotropy and shear-layer edge dynamics in the net kinetic energy decay phase.

The turbulence decay is associated with a negative rate of shear production in parts of the turbulent layer, implying kinetic energy transfer from the turbulence to the mean flow. It is found that momentum flux reversal, as well as heat flux reversal, is present in parts of the domain. This is related to a change of roles of shear and buoyancy production of turbulence kinetic energy, with the former actually transferring energy into the mean flow field, which may lead to relaminarization.

The results indicate that the faster increase of small-scale anisotropy relative to large-scale anisotropy, reported earlier ${ }^{2}$ for shear-free stratified flow, also holds in the case of sheared stratified flow. However, the anisotropy of thermal dissipation (which is also a measure of small-scale anisotropy) differs significantly from that of viscous dissipation; the second invariant of the former exceeds that of the latter.

Curiously, the present analysis shows that the Reynolds stress anisotropy increases only up to a certain level of stratification, corresponding roughly to $\mathrm{Ri}_{g} \approx 0.4$. For even stronger stratification, the anisotropy begins to decrease. It has been shown that a likely explanation for this is relaminarization.

The coherent structures of the large-scale turbulence are generally cylindrical in the center of the shear layer. In the layer edge, the structures become increasingly compressed into ellipsoids with time. The Reynolds stresses are highly $1 \mathrm{C}$ in the entire layer, except for a narrow region at the layer edge, where it turns $2 \mathrm{C}$ as stratification increases. The region of $2 \mathrm{C}$ Reynolds stresses corresponds to the region where $\mathrm{Ri}_{g} \gtrsim 0.4$. The variation of Reynolds stress anisotropy across the layer has important consequences for dispersion modeling; Gaussian dispersion models commonly utilize the velocity variance to estimate the deformation of contaminant clouds. ${ }^{30}$ The variance is thus important for estimating the spread of a Gaussian puff as well as the decay of peak concentration. Additionally, the dimensionality tensor could be used to improve dispersion modeling by incorporating the state of large-scale structures.

As turbulence decays, the separation between the large and small velocity time scales is reduced in the inner part of the shear layer. Closer to the layer edge, the large time scale - and hence the scale separation - increases. In the present study, a time scale suitable for thermal dissipation is proposed. The thermal scale separation increases in the layer edge, but it remains the same in the center region throughout the decay phase. Stratification has a more profound effect on the larger scales, and it seems to affect the thermal scales more strongly than the momentum scales.

A mechanical to thermal time scale ratio of $\mathcal{R} \approx 0.85$ is observed in the shear-dominated region, i.e., approximately half of the reported value ${ }^{24}$ for shear-free flow with a uniform temperature gradient.

The dissipation anisotropy and pressure-strain components are highly reminiscent of how they behave near solid walls. The latter, in particular its association with a change of sign of $\phi_{33}$, suggests that the imposition of strong stable stratification mimics the non-local pressure effects of an impenetrable wall. On the other hand, the Reynolds stresses show little resemblance to their near-wall behavior, which implies that the kinematic blocking effect of walls is not emulated by the imposed stable stratification. Consequently, turbulence models employed in stably stratified flows ought to be non-local to incorporate important effects of the stratification. Since the dimensionality tensor resembles its near-wall behavior, models based on this non-local single-point quantity might be beneficial.

In conclusion, there are important indicators of a significant change in the dynamics and kinematics near the strongly inhomogeneous KH layer edge, which has implications for predictive 
turbulence model formulations. For example, large dissipation anisotropies generally imply that the local isotropy assumption is invalid, meaning that common dissipation rate models may not suffice. Moreover, local pressure-strain models may need to account for non-local effects, cf., e.g., Durbin and Speziale. ${ }^{31}$

\section{ACKNOWLEDGMENTS}

This work has been funded in part by the Research Council of Norway, through Project No. NFR-214881.

${ }^{1}$ W. D. Smyth and J. N. Moum, "Anisotropy of turbulence in stably stratified mixing layers," Phys. Fluids (1994-present) 12, 1343-1362 (2000).

${ }^{2}$ S. Thoroddsen and C. Van Atta, "The influence of stable stratification on small-scale anisotropy and dissipation in turbulence,” J. Geophys. Res.: Oceans (1978-2012) 97, 3647-3658, doi:10.1029/91JC02727 (1992).

${ }^{3}$ J. Kim, P. Moin, and R. Moser, "Turbulence statistics in fully developed channel flow at low Reynolds number," J. Fluid Mech. 177, 133-166 (1987)

${ }^{4}$ J. L. Lumley, “Computational modeling of turbulent flows,” Adv. Appl. Mech. 18, 123-176 (1979).

${ }^{5}$ S. C. Kassinos, W. C. Reynolds, and M. M. Rogers, "One-point turbulence structure tensors," J. Fluid Mech. 428, 213-248 (2001).

6 J. Pereira and J. Rocha, "Prediction of stably stratified homogeneous shear flows with second-order turbulence models," Fluid Dyn. Res. 42, 045509 (2010).

${ }^{7}$ J. Werne, T. Lund, and D. Fritts, "CAP phase II simulations for the air force HEL-JTO project: Atmospheric turbulence simulations on NAVO's 3000-processor IBM P4+ and ARL's 2000-processor intel xeon EM64T cluster," in (IEEE, 2005), pp. 100-111.

${ }^{8}$ M. Lee and W. Reynolds, "Numerical experiments on the structure of homogeneous turbulence," Thermosciences Division Report No. TF-24, 1985.

${ }^{9}$ K. Julien, S. Legg, J. McWilliams, and J. Werne, “Rapidly rotating turbulent Rayleigh-Bénard convection,” J. Fluid Mech. 322, 243-273 (1996).

${ }^{10}$ P. R. Spalart, R. D. Moser, and M. M. Rogers, "Spectral methods for the Navier-Stokes equations with one infinite and two periodic directions," J. Comput. Phys. 96, 297-324 (1991).

${ }^{11}$ B. Reif, J. Werne, Ø. Andreassen, C. Meyer, and M. Davis-Mansour, "Entrainment-zone restratification and flow structures in stratified shear turbulence," in Proceedings of the Summer Program (Center for Turbulence Research, Stanford University, 2002).

${ }^{12}$ D. C. Fritts, T. L. Palmer, Ø. Andreassen, and I. Lie, "Evolution and breakdown of Kelvin-Helmholtz billows in stratified compressible flows. Part I: Comparison of two- and three-dimensional flows," J. Atmos. Sci. 53, 3173-3191 (1996).

${ }^{13}$ T. L. Palmer, D. C. Fritts, and Ø. Andreassen, "Evolution and breakdown of Kelvin-Helmholtz billows in stratified compressible flows. Part II: Instability structure, evolution, and energetics," J. Atmos. Sci. 53, 3192-3212 (1996).

${ }^{14}$ S. Thorpe, "On the Kelvin-Helmholtz route to turbulence," J. Fluid Mech. 708, 1-4 (2012).

15 W. D. Smyth and J. N. Moum, "Ocean mixing by Kelvin-Helmholtz instability," Oceanography 25, 140-149 (2012).

${ }^{16}$ J. W. Miles, “On the stability of heterogeneous shear flows," J. Fluid Mech. 10, 496-508 (1961).

${ }^{17}$ J. Rohr, E. Itsweire, K. Helland, and C. Van Atta, "Growth and decay of turbulence in a stably stratified shear flow," J. Fluid Mech. 195, 77-111 (1988).

${ }^{18}$ J. Dougherty, "The anisotropy of turbulence at the meteor level," J. Atmos. Terr. Phys. 21, 210-213 (1961).

${ }^{19}$ R. Ozmidov, "On the turbulent exchange in a stably stratified ocean," Izv., Acad. Sci., USSR, Atmos. Oceanic Phys. 1, 861-871 (1965), available at http://www.ocean.ru/index2.php?option=com_docman\&task=doc_view\&gid=598\& Itemid=78.

${ }^{20}$ B. P. Reif and Ø. Andreassen, “On local isotropy in stratified homogeneous turbulence," SIAM J. Appl. Math. 64, 309-321 (2003).

${ }^{21}$ R. W. Johnson, Handbook of Fluid Dynamics (CRC Press, 1998).

${ }^{22}$ M. Garcia-Villalba and J. C. Del Alamo, "Turbulence modification by stable stratification in channel flow," Phys. Fluids (1994-present) 23, 045104 (2011).

23 J. C. Wyngaard, Turbulence in the Atmosphere (Cambridge University Press, 2010).

${ }^{24}$ A. Sirivat and Z. Warhaft, "The effect of a passive cross-stream temperature gradient on the evolution of temperature variance and heat flux in grid turbulence," J. Fluid Mech. 128, 323-346 (1983).

25 J. Weinstock, "Energy dissipation rates of turbulence in the stable free atmosphere," J. Atmos. Sci. 38, 880-883 (1981).

${ }^{26}$ H. Fossum, E. Wingstedt, and B. Reif, "A model for the viscous dissipation rate in stably stratified, sheared turbulence," Geophys. Res. Lett. 40, 3744-3749, doi:10.1002/grl.50663 (2013).

${ }^{27}$ P. A. Durbin and B. P. Reif, Statistical Theory and Modeling for Turbulent Flows (John Wiley \& Sons, 2011).

${ }^{28}$ R. D. Moser, J. Kim, and N. N. Mansour, "Direct numerical simulation of turbulent channel flow up to Re $=590$," Phys. Fluids 11, 943-945 (1999).

${ }^{29}$ B. E. Launder and N. Shima, "Second-moment closure for the near-wall sublayer-development and application," AIAA J. 27, 1319-1325 (1989).

30 J. D. Wilson and B. L. Sawford, "Review of Lagrangian stochastic models for trajectories in the turbulent atmosphere," Boundary-Layer Meteorol. 78, 191-210 (1996).

${ }^{31}$ P. Durbin and C. Speziale, "Local anisotropy in strained turbulence at high Reynolds numbers," J. Fluids Eng. 113, 707-709 (1991). 